\title{
Preface on the Special Issue Global Optimization with Multiple Criteria: Theory, Methods and Applications
}

\author{
Kaisa Miettinen ${ }^{1}$. Francisco Ruiz ${ }^{2}$ \\ Published online: 6 September 2019 \\ (c) The Author(s) 2019
}

In the field of operations research, the task of searching for global optima faces complex challenges. Two of these challenges constitute the main motivation of this special issue:

- Many real optimization applications deal with making decisions in the presence of several conflicting objectives and different methodologies have been developed to solve these so-called multiobjective optimization problems. In general, they involve either the determination of a representative set of Pareto optimal solutions, or supporting the search for the decision maker's most preferred solution. In both cases, many of the existing methodologies imply solving a series of single objective optimization problems which are built from the original multiobjective one. Besides, in some of the methods, these problems need to be solved in real time during an interaction with the decision maker and thus, in reasonable times.

- The features of real problems may result in optimization problems with a growing complexity in terms of the number of variables and constraints considered, as well as in terms of the types of variables. This leads to considering problems with many integer/binary variables and/or highly nonconvex and even nondifferentiable functions. This calls for new methodologies designed to find global solutions (or at least, good approximations of them) in manageable times. In particular, many metaheuristic methods (like evolutionary approaches) have been developed, and they have proved to be able to tackle problems that cannot be solved with classical (exact) optimization methods.

The joint consideration of these two challenges, that is, facing multiobjective optimization problems with highly complex features, results in an exponential growth of complexity. As said, solving multiobjective optimization problems usually involves solving many single objective subproblems, whose complexity is at least as high as that of the original problem. Besides, the frequent need to obtain such solutions in real time adds the challenge of producing them as quickly as possible. Therefore, the development of theoretical and algorithmic aspects

Kaisa Miettinen

kaisa.miettinen@jyu.fi

Francisco Ruiz

rua@uma.es

1 University of Jyvaskyla, Faculty of Information Technology, P.O. Box 35 (Agora), FI-40100 University of Jyvaskyla, Finland

2 Department of Applied Economics (Mathematics), Universidad de Málaga, Calle Ejido 6, 29071 Málaga, Spain 
for this type of problem, as well as their application to real decision processes, have been the basic guidelines of this special issue.

The origin of this issue was the 24th International Conference on Multiple Criteria Decision Making (MCDM 2017) held in Ottawa, Canada, in July, 2017. This series of conferences is organized every two years by the International Society on MCDM (www.mcdmsociety. org). This volume accepted expanded versions of some of the papers presented at the conference, as well as other submissions prepared by researchers who did not attend the conference. We received 23 submissions, among which 3 were finally accepted for publication after a rigorous reviewing process. In fact, each paper was reviewed by at least three reviewers. We would like to thank the reviewers for their selfless and valuable collaboration and all researchers who submitted papers for this special issue. Besides, we would also like to thank the Journal of Global Optimization, and especially its editor-in-chief, Prof. Sergiy Butenko, for their help and support throughout the whole editing process of this special issue.

In the article "Efficient Computation of Expected Hypervolume Improvement Using Box Decomposition Algorithms", Kaifeng Yang, Michael Emmerich, Andre Deutz and Thomas Bäck concentrate on multiobjective Bayesian global optimization. They utilize Gaussian process models and propose an efficient algorithm for calculating expected hypervolume improvement, which uses hyperbox decomposition and is not limited to two or three objectives.

Ozgu Turgut, Evrim Dalkiran and Alper E. Murat consider multiobjective optimization problems with integer variables in their article "An Exact Parallel Objective Space Decomposition Algorithm for Solving Multi-objective Integer Programming Problems". They propose a deterministic and exact algorithm which applies decomposition and scalarization. The authors demonstrate the performance of the algorithm with test problems with up to six objective functions.

The third article "Multi-Objective Unconstrained Combinatorial Optimization: A Polynomial Bound on the Number of Extreme Supported Solutions" by Britta Schulze, Kathrin Klamroth and Michael Stiglmayr was already published in volume 74, issue 3 of this journal.

In addition, a fourth paper was added to the special issue after it was accepted as a regular submission, due to fit of the topic to the theme of the special issue. In this article, named "Nonmonotone Line Searches for Unconstrained Multiobjective Optimization Problems", Kanako Mita, Ellen Hidemi Fukuda and Nobuo Yamashita consider two types of nonmonotone line searches for descent methods and propose a new nonmonotone technique specifically for multiobjective optimization problems.

Acknowledgements Open access funding provided by University of Jyväskylä (JYU).

Open Access This article is distributed under the terms of the Creative Commons Attribution 4.0 International License (http://creativecommons.org/licenses/by/4.0/), which permits unrestricted use, distribution, and reproduction in any medium, provided you give appropriate credit to the original author(s) and the source, provide a link to the Creative Commons license, and indicate if changes were made.

Publisher's Note Springer Nature remains neutral with regard to jurisdictional claims in published maps and institutional affiliations. 